\title{
EOP:
}

\section{Monitoreo ambulatorio de la presión arterial y riesgo cardiovascular}

Kopitowski K. Monitoreo ambulatorio de la presión arterial y riesgo cardiovascular. Evid. actual. práct. ambul; 9(3): 92-93, May-Jun 2006.

\section{Escenario clínico:}

Un médico de familia atiende a una mujer de 65 años con diagnóstico de hipertensión arterial realizado un año antes. La mujer no recibe medicación y tiene varios registros de presión arterial (PA) tanto en el Centro de salud como en el domicilio. El promedio de los controles es de $155 \mathrm{~mm} \mathrm{Hg}$ de tensión arterial sistólica (TAS) y103 $\mathrm{mmHg}$ de tensión arterial diastólica (TAD). Sin embargo, existe una enorme dispersión en los valores y ese promedio es el resultado de valores que oscilan entre 170 y $120 \mathrm{mmHg}$ de TAS y 110 y $85 \mathrm{mmHg}$ de TAD. El médico recuerda que existe un estudio, el monitoreo ambulatorio de la presión arterial (MAPA); que tiene indicaciones muy puntuales y se pregunta si realizarlo en esta paciente podría aportar datos pronósticos acerca del verdadero riesgo de eventos cardiovasculares a futuro.

\section{Pregunta que generó el caso:}

En pacientes hipertensos (población), los datos arrojados por el MAPA (exposición) vs los valores de toma de TA convencionales (comparación) predicen eventos cardiovasculares con mayor precisión (resultado clínico de interés)?

\section{Estrategia de búsqueda:}

Se realizó una búsqueda en TRIP database (www.tripdatabase.com) empleando como palabra clave "ambulatory blood pressure monitoring". Entre los resultados se encontró una cita de una publicación secundaria que lucía atractiva y disponible a texto completo gratuitamente:

Powers, Donald V Review: ambulatory blood pressure monitoring predicts clinical outcomes Evid Based Med 2003 8: 120

Dado que la cita era de 2003, se decidió completar la búsqueda en MEDLINE usando como término MeSH Blood Pressure Monitoring, Ambulatory y limitando para estudios pronósticos en los últimos 5 años en población adulta.

\section{Varias citas lucían atractivas}

Hansen TW, Jeppesen J, Rasmussen S, Ibsen H, Torp-Pedersen C. Ambulatory blood pressure monitoring and risk of cardiovascular disease: a population based study.

Am J Hypertens. 2006 Mar;19(3):243-50.

Clement DL; De Buyzere ML; De Bacquer DA. Prognostic value of ambulatory blood-pressure recordings in patients with treated hypertension.

N Engl J Med. 2003 Jun 12;348(24):2407-15

\section{El monitoreo ambulatorio continuo de presión arteria}

En las últimas décadas, las técnicas para medir la presión arterial para determinar si un paciente es hipertenso o no, han sufrido cambios sustanciales. La construcción del conocimiento acerca de los riesgos de la hipertensión arterial (HTA) y los beneficios de su tratamiento ha surgido de obtener un número pequeño de lecturas de TA con el método auscultatorio en el ámbito de la consulta médica. Sin embargo, estas mediciones que tienen enorme valor poblacional, pueden no estimar adecuadamente el riesgo individual por diferentes razones, entre las que se pueden mencionar una técnica pobre, el efecto de "guardapolvo blanco" y la variabilidad inherente de la TA.

Todas las mediciones clínicas de la TA deben considerase subrogantes de la "verdadera" presión arterial del paciente que podría definirse como la TA promedio en largos períodos de tiempo.
Existen dos maneras de mejorar la estimación de la verdadera presión arterial: el MAPA y el automonitoreo por parte del paciente. EI MAPA empela una técnica oscilométrica. Consiste en un dispositivo que el paciente lleva durante 24 hs mientras realiza su actividad diaria. Los aparatos están programados para tomar la TA cada 15 o 30 minutos. Al final del período de evaluación, los resultados se vuelcan a una computadora que analiza las lecturas. El informe arroja tres datos: la TA promedio, la variabilidad de la TA y el ritmo circadiano. El punto de corte para la TA en el MAPA es $135 / 85$ ya que se corresponde con un valor de consultorio de 140/90.

El "JNC 7" (Seventh Report of the National Committee on Prevention, Detection, and treatment of High Blood Pressure) y la WHO-ISH (Word Health Organization-International Society of Hypertension) recomiendan el empleo del MAPA ante la sospecha de HTA de guardapolvo blanco, HTA lábil, HTA resistente, o episodios hipotensivos.

\section{Resumen de la evidencia}

Clement DL; De Buyzere ML; De Bacquer DA. Prognostic value of ambulatory blood-pressure recordings in patients with treated hypertension. N Engl J Med. 2003 Jun 12;348(24):2407-15.

Este estudio quiso evaluar si el MAPA predice eventos cardiovasculares independientemente de las mediciones obtenidas por el médico en el consultorio y los factores de riesgo convencionales en pacientes hipertensos tratados. Los investigadores realizaron un estudio de cohortes en 1963 pacientes con un seguimiento de 5 años. La variable independiente* fue el MAPA basal y la variable dependiente* fue "eventos cardiovasculares".

Hubo 157 pacientes con eventos. En el análisis multivariable* que ajustó por edad, sexo, tabaquismo, diabetes mellitus, nivel de colesterol, índice de masa corporal, historia de eventos cardiovasculares y TA medida en el consultorio médico; se evidenció que valores promedio más altos en el MAPA fueron predictores independientes de nuevos eventos. EI RR ajustado de eventos cardiovasculares asociados a cada desvío estándar de aumento de la TA sistólica en el MAPA fue de 1,34 (IC 95\% 1,11-1,62). En el caso de la TA diastólica fue de 1,21 (IC 95\% 1,01-1,46).

Cuando los valores del MAPA se evaluaron en mayores de 135 $\mathrm{mmHg}$ de TAS vs menores de dicha cifra el RR para eventos cardiovasculares fue 1,75 (IC 95\% 1,15-2,63).

Los autores concluyeron que en los pacientes con HTA tratada, una TA diastólica o sistólica elevadas medidas por MAPA predicen eventos aún luego de ajustar por factores de riesgo clásicos, incluida la TA medida por el médico.

Hansen TW, Jeppesen J, Rasmussen S, Ibsen H, Torp-Pedersen $C$. Ambulatory blood pressure monitoring and risk of cardiovascular disease: a population based study. Am J Hypertens. 2006 Mar;19(3):243-50.

Los autores quisieron evaluar la relación entre el MAPA y la mortalidad cardiovascular. Para ello realizaron un estudio de cohortes en el que se incluyó una muestra aleatoria de 1700 hombres y mujeres daneses entre 41 y 72 años libres de enfermedades cardiovasculares.

Al inicio del estudio se recabó información sobre factores de riesgo y se registró el resultado del MAPA y de la TA medida en el consultorio. Tanto la información del MAPA como de las mediciones con esfingomanómetro estaban adecuadamente estandarizadas. El estudio siguió a los participantes por 9.5 años.

Durante el estudio, murieron 174 participantes (el $36 \%$ de causas cardiovasculares).

En un modelo multivariable* que ajustó por factores de riesgo el RR para mortalidad cardiovascular asociado con $10 \mathrm{mmHg}$ de incremento en la TA sistólica y $5 \mathrm{~mm} \mathrm{Hg}$ en la diastólica fue de 1,51 (IC95\% 1,27-1,77) y $1,43(1,26-1,71)$ respectivamente. Para el resultado de mortalidad global, los RR fueron $1,18(1,06-1,31)$ y $1,18(1,09-1,28)$. Los RR para mortalidad cardiovascular fueron 
menores para la TA en el consultorio y la TA en el consultorio no predijo mortalidad global. Cuando se incluye la TA en el consultorio y los resultados del MAPA en el mismo modelo multivariable, sólo el MAPA resultó ser predictor de la mortalidad global y cardiovascular.

Este estudio encontró relación lineal entre MAPA y mortalidad, reforzando la idea de que no existe un umbral protector.

Los autores concluyeron que el MAPA provee información pronóstica más allá de la que proporciona la TA medida en el consultorio.

\section{Conclusión y comentario}

Si bien para la pregunta que generó el caso la respuesta pareciera ser que el MAPA es mejor predictor de eventos que el registro convencional, no existen ensayos que hayan probado que una estrategia basada en el MAPA es superior que una estrategia "convencional" en lograr mejor pronóstico de los pacientes.

Probablemente, pueda lograrse una disminución de la brecha entre la predicción de los registros en el consultorio vs. el MAPA obteniendo más registros de TA por consulta y haciendo un promedio". Un estudio evaluó diferencias en el manejo de los pacientes acorde a si el tratamiento era guiado por valores de consultorio o MAPA ${ }^{2}$. Dicho estudio aleatorizado encontró que el nivel de TA alcanzado fue el mismo. Las diferencias halladas entre ambas ramas fueron: menor requerimiento de número de fármacos (para igual control) en el grupo MAPA y mayor número de pacientes que pudieron suspender tratamiento en la rama MAPA (26\% vs $7 \%$ ) sugiriendo una prevalencia de $19 \%$ de HTA de guardapolvo blanco, similar a la reportada en la literatura.
Sin embargo, el empleo rutinario del MAPA está limitado por múltiples razones que incluyen la falta de disponibilidad de los equipos, el costo y la cobertura de la práctica por las Obras sociales y prepagas.

Por este motivo, se ha prestado mayor atención a las tomas de TA domiciliarias a través de dispositivos semiautomáticos. Estas tomas en el hogar o el trabajo correlacionan más con el MAPA que las mediciones en el consultorio ${ }^{3}$ y son mejores predictores de accidentes cerebrovasculares ${ }^{4}$.

Si bien las mediciones en la casa pueden tener error sustancial, el mismo puede minimizarse con entrenamiento apropiado y revisión del dispositivo para verificar la precisión. Los beneficios de este tipo de control incluyen la identificación de la HTA de guardapolvo blanco, evaluación de respuesta a las drogas y mayor adherencia al tratamiento ${ }^{5-6}$. Toda esta discusión, no debe hacernos perder de vista que el problema en el diagnóstico y tratamiento de la HTA sigue siendo que la mayor parte de los hipertensos no sabe que lo es, que la mayoría no se trata y de los que se tratan pocos llegan a la meta. Por lo tanto, las prioridades en el abordaje de la HTA deben estar puestos en el reconocimiento, tratamiento y control de los hipertensos.

No obstante, frente al caso individual, podemos considerar (por el momento) las siguientes indicaciones para indicar un MAPA:

- Sospecha de HTA guardapolvo blanco

- Sospecha de HTA episódica

- HTA resistente

- Sospecha de episodios hipotensivos

- Disfunción autonómica.

Dra. Karin Kopitowski [ Unidad de Medicina Familiar y Preventiva. Hospital Italiano de Buenos Aires. ]

Referencias

1. Fagard, RH, Staessen, JA, Thijs, L. Prediction of cardiac structure and function by repeated clinic and ambulatory blood pressure. Hypertension $1997 ; 29: 22$. 2. Staessen, JA, Byttebier, G, Buntinx, F, et al. Antihypertensive treatment based on conventional or ambulatory blood pressure measurement. A randomized controlled trial. Ambulatory Blood Pressure Monitoring and Treatment of Hypertension Investigators. JAMA 1997; 278:1065.

3.Verberk, WJ, Kroon, AA, Kessels, AG, de Leeuw, PW. Home Blood Pressure Measurement A Systematic Review. J Am Coll Cardiol $2005 ; 46: 743$.

4.Asayama, K, Ohkubo, T, Kikuya, M, et al. Prediction of stroke by self-measurement of blood pressure at home versus casual screening blood pressure measurement in relation to the Joint National Committee 7 classification: the Ohasama study. Stroke 2004; 35:2356.

5.Krakoff, LR. Cost-effectiveness of ambulatory blood pressure: a reanalysis. Hypertension 2006; 47:29.

6. Rickerby, J. The role of home blood pressure measurement in managing hypertension: an evidence-based review. J Hum Hypertens $2002 ; 16: 469$.

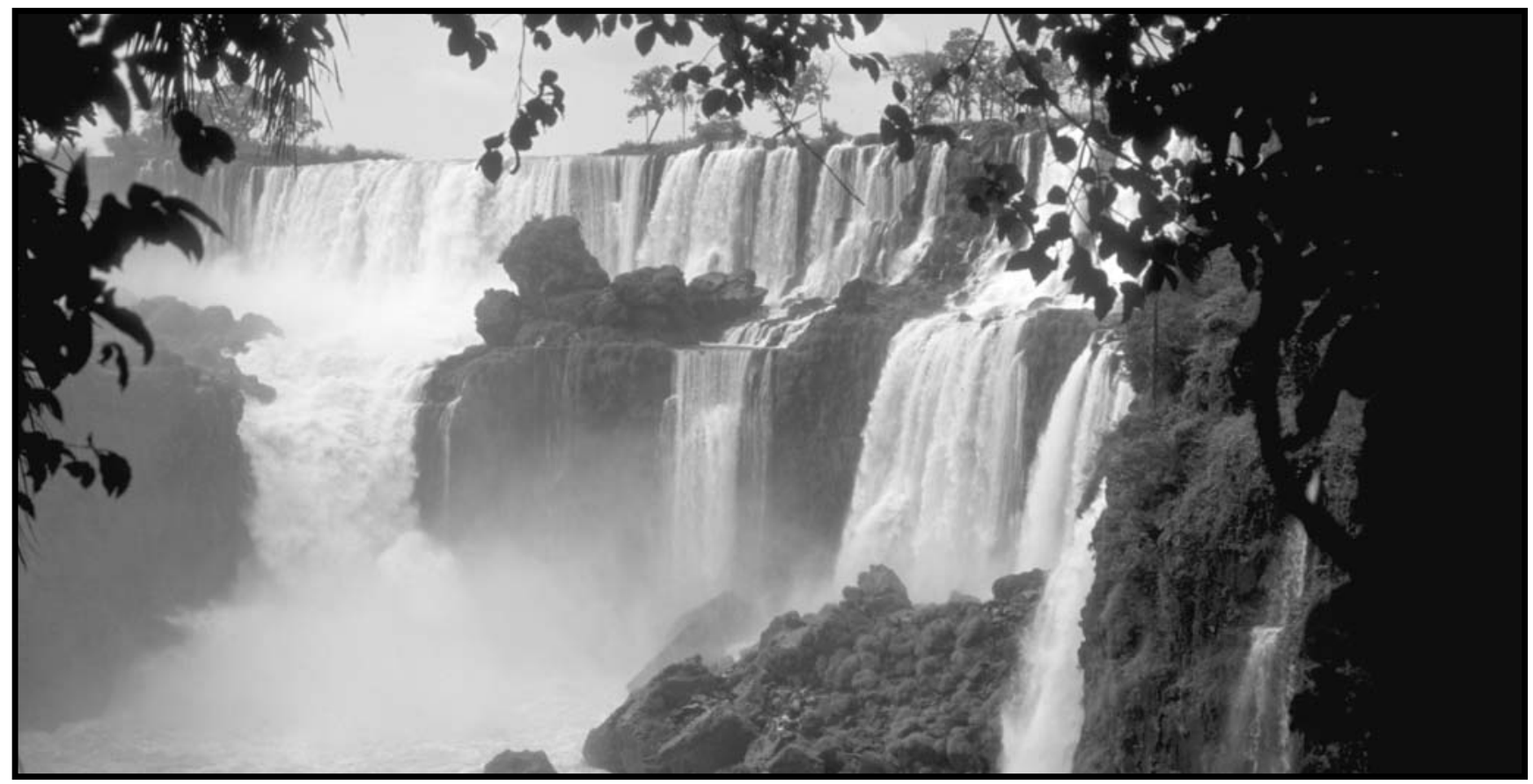

